\title{
Determination of transient thermal characteristics for thermal electric behavioral models of integrated circuits
}

\author{
Drakin A. Yu., Potapov L. A., Shkolin A. N. \\ Bry ansk State Technical University, Russia
}

\begin{tabular}{l}
\hline \hline Article Info \\
\hline Article history: \\
Received Aug 21, 2019 \\
Revised Nov 12, 2019 \\
Accepted Feb 10, 2020 \\
\hline
\end{tabular}

\section{Keywords:}

Integrated circuits

Matlab

P-n-junction

Thermal resistance

Thermoelectric model

\begin{abstract}
In the current study, it was tried to describe a method for determining thermal characteristics of integrated micro-circuits to identify thermal parameters of multidisciplinary (thermal-electric) behavioral models. The problem is solved on the example of high-frequency pulse voltage converters. A solution was proposed to refine the minimum structure of the thermoelectric model based on an iterative least squares method using the Levenberg-Marquardt algorithm, as well as a graph of the spectral den-sity of time constants. This made it possible to reduce the influence of the filtering factor in the deconvolution operation when building a thermal model using the structural function of the thermal characteristic transition. Also, the results obtained can be used to build integrated circuits (IC) behavioral models, taking into account the thermal processes occurring in them.
\end{abstract}

This is an open access article under the $\underline{C C B Y-S A}$ license.

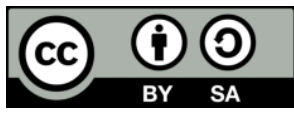

\section{Corresponding Author:}

Drakin A. Yu.,

Bryansk State Technical University, Russia.

Email: bakhteeyar@gmail.com

\section{INTRODUCTION}

Integrated circuits (IC) of high-frequency switching voltage converters (HFSVC) are widely used in power supplies of any electronic devices that make up the onboard systems of aircraft and spacecraft, household appliances, computers, mobile gadgets, etc. The area of their use as regulators of constant voltage is also constantly expanding due to the development of areas where linear constant-voltage regulators of the compensation type were used previously.

Under modern conditions, shortening the development time of electronic devices is impossible without modeling. When developing the design of power electronics devices, one of the important tasks of modeling is to take into account the mutual influence of the thermal and electrical characteristics of semiconductor devices and microcircuits, which affects the reliability and performance of the electron ic device as a whole. Therefore, the practice of applying complex multidisciplinary models is expanding. Usually, finite element models with distributed parameters [1-5] or with lumped parameters [6-9] are used. The first type of models allows modeling, taking into account many design features, but it requires a larger amount of input data for model compilation as well as a high level of scientific competence of the developer, which is not often observed in engineering practice. In this regard, the second type of the model has a number of advantages. Such models of integrated circuits can be successfully implemented in the popular electronic industry languages SPICE, VHDL-AMS, Verilog-A, etc. [9].

The full-fledged synthesis of multidisciplinary behavioral models of IC, based on the analysis of the electrical circuit and the design of the core of the chip, is an independent task and is beyond the scope of this article. The authors' task is to find and substantiate a method for determining the IC thermal characteristics, which allows it to be used in the synthesis of multidisciplinary behavioral models of high- 
frequency impulse-voltage chip circuits, taking into account the features inherent in a particular HFSVC design. This paper solves the problem of obtaining a model describing the HFSVC -thermal properties with respect to the experimentally measured thermal transient response (TTR) the dependence of the crystal (junction) temperature of the circuit on time $\mathrm{TJ}(\mathrm{t})$.

\section{LITERATURE REVIEW}

To determine the TTR in the standard [10], it is recommended to periodically connect the semiconductor device under test to the nominal mode with a frequency of less than $66.7 \mathrm{~Hz}$, to connect a small measuring current between pulses in the pauses of $100 \mu$ s as well as measure the UF forward voltage at the p-n-junction. Using the UF (TJ) calibration, it is possible to obtain a set of TJk transition temperature values at the corresponding tk time points or a graph of the nonlinear TJk (tk) dependence.

In the experimental TTR determination, direct measurement of the crystal temperature is impossible. The placement of the thermocouple in the instrument case (in close proximity to the crystal) or on the case [11] leads to a distortion of the real picture of the thermal processes taking place in the semiconductor device. In this regard, the methods of indirect determination of the crystal temperature of the device, based on the control of temperature-dependent parameters (TDP) inherent in the device under test, are actively used. The direct current voltage drop on the TJ $\mathrm{p}-\mathrm{n}$ junction, the transconductance of the transistor $\mathrm{S}$, reverse current (IRR) and the characteristics of the reverse pn junction recovery process (tRR, IRR,max), signal delay-propagation time tD [12-14] are usually used as IC TDP. When choosing a TDP HFPVC circuits, the following conclusions can be made. Due to the lack of connection from the external leads to the input circuit of the power transistor as part of the chip, it is not possible to use the transistor slope as the TDP.

The use of tRR, IRR, max, tD dynamic parameters as a TDP makes it possible to experimentally determine the TTR of microcircuits directly in the nominal mode of the microcircuit; - the measurement of heating power requires the dynamic measurement of the microcircuit power consumption and the value of its "transit component" attributable to passive elements (choke, capacitors, external power diode) as well as load included in a typical circuit switching on HFSVC.

If TJ is used as a TDP, then usually difficulties in determining the TTR in the nominal mode of the chip are also associated with the implementation of heating power dynamic measurement, and additionally with the HFSVC tss start-up time, which is fractions and milliseconds and limits the resolution to select the minimum pause time during which the measuring current is applied to determine the UF at the p-n-junction.

Recently, methods for determining the parameters of the electric-thermal model [15] are being developed, based on the analysis of frequency thermal characteristics, that is, the dependence of the amplitude and phase of change of the transition temperature TJ $(\omega)$ on the frequency when the device is heated with a power varying harmonically $\mathrm{P}(\mathrm{t})=\mathrm{P} 0+\mathrm{Pmsin} \omega \mathrm{t}$. In them, based on the calculation of the amplitudes and phases of the main harmonics of the heating power and temperature of the $p-n$ junction, the module of transient thermal resistance (thermal impedance) and the $\varphi$ phase shift between the temperature of the $\mathrm{p}-\mathrm{n}$ junction and heating power is determined. Next, the dependence of the thermal impedance module on heating power modulation frequency $Z \operatorname{thjc}(\omega)$ and the thermal circuit parameters are determined. By analogy with electrical circuits, thermal impedance is considered as a complex number Zthjc $=$ Zthjcej $\varphi$, the real part of which determines Rthjc=Re[Zthjc ] thermal resistance.

A technical solution: "Thermal Transient tester T3Ster" [16], which implements the methods of standards $[11,17]$ used to control the thermal resistance of semiconductor devices and integrated circuits is known. The device provides the ability to determine the direct voltage drop at the p-n-junction at a microsecond time resolution after the end of the heating pulse. Based on these data, using the software, the tested chip thermal resistance as a whole and of individual links of the thermal circuit are calculated. The TTR curve analysis obtained by any method is usually performed by classical methods, for example, by analyzing the first and second derivatives [12].

Recently, when analyzing the TTR curve, the apparatus of structure functions [16, 18-20] has been often used, which allows determining thermal resistance components. In accordance with it, at the first stage, using the inverse convolution of the graph of the first TTR derivative in the logarithmic time $s c a l e ~ z=\ln (t)$ and the weight function $w z=\exp (\mathrm{z}-\exp (\mathrm{z}))$ the dependence of the spectral density of time constants (TCSD) is built. Next, it is discretized with a small dz step and a thermal model is constructed according to the Foster scheme. At the second stage, this scheme is transformed into the Cauer scheme, for example, according to the method in [2], and on its basis a set of dependences of the heat capacities on the thermal resistances of the elements included in the resulting scheme is obtained. This dependency is called the structure function, since for a one-dimensional heat flow, it characterizes the layers that make up the design of the sample under 
study. This is manifested in abrupt changes in the graph of the structure function, and is easily determined on the graph of the first derivative of this function (the differential structure function).

The method of structure functions is constantly refined and supplemented [21-24]. Critical factors for it are the noise level, which is always present in the TTR experimental determination and the need to analyze relatively small values of thermal characteristics, which may result in "peak emissions" in the beginning of the structural function graph, which in turn happens due to the influence of the first of the above mentioned factors. The use of well-known methods of filtering signals [16, 25] to reduce the influence of the first factor on one hand additionally requires the selection of a filter, and on the other hand leads to a distortion of the real picture of the occurring thermal processes.

In [26-29], the authors used the original method of thermal relaxation spectrometry to analyze TTR. In [27], the thermal parameters of high-power bipolar transistors in plastic cases TO-252 and TO-126 were estimated using thermal relaxation differential spectrometry. For this purpose, a relaxation impedance spectrometer developed by the authors [28] was used, which makes it possible to determine the TTR. The thermal constants of the instruments and the distribution of the thermal resistance structure are determined in the form of discrete and continuous spectra. The continuous spectrumis calculated on the basis of higher-order derivatives of dynamic thermal impedance and corresponds to the Foster model, the discrete one corresponds to the Cauer model. The structure of the thermal resistance of the devices was represented in the form of a six-link electric-thermal RC model.

\section{METHODOLOGY AND MATERIALS}

For the experimental TTR determination, a method that does not require periodic connection of the test HFSVC IC [10] was tested. The crystal is heated by a direct current passing through a power diode located on the crystal and connected in parallel with the power transistor. Measurement of the crystal temperature is performed by measuring the forward UF voltage at another $\mathrm{p}-\mathrm{n}$ junction at a small measuring current and then converting UF voltage to TJ temperature, using the TJ (UF). The resulting T(t) dependence can be approximated by several exponents.

$$
T_{J}(t)=A_{T}\left(1-e^{p_{1 T} t}\right)+B_{T}\left(1-e^{p_{2 T} t}\right)
$$

The transient thermal characteristic can be determined using application software and a tester [9], which is designed to control the electrical parameters of high-frequency switching voltage converters (HFSVC) under mass production conditions. As a result of the UF(t) experiment, an array of digits is obtained, which should be converted into an array of digits $T \sum(\mathrm{t})$ and then $\mathrm{T}(\mathrm{t})$, где $\mathrm{T}(\mathrm{t})=\mathrm{T} \sum(\mathrm{t})-\mathrm{T} \alpha$, $\mathrm{T}$ is the heating temperature of the HFSVC crystal; $\mathrm{T} \sum$ is the total HFSVC crystal temperature; $\mathrm{T} \alpha$ is the ambient temperature.

The next step is the process of approximation of the experimental TTR. Approximate TTR can be a different number of exponents. The authors used the Matlab tools [30]. To find the approximate TTR dependence in Matlab, the least squares method is implemented using the Leven-Berg-Marquardt optimization algorithm. To approximate it is necessary to perform the following operations:

a. To convert the experimentally obtained matrix $\mathrm{UF}(\mathrm{t})$ to $\mathrm{TJ}(\mathrm{t})$, using a previously plotted temperature coefficient of voltage coefficient for a heat-sensitive parameter (TDP), which is one of the internal p-n-transitions of the microchip crystal. It also recalculates the heating power of the crystal temperature array to $1 \mathrm{~W}$, taking into account the known value of the stabilized heating power.

b. To set the type of approximating dependence. For example, for an up-proxy dependence for an independent time variable $\mathrm{x}$ based on six exponentials with 12 sought-for coefficients (A, B, C, D, E, F, $\mathrm{p} 1, \mathrm{p} 2, \mathrm{p} 3, \mathrm{p} 4)$, its appearance is as follows:

$$
F(x)=A\left(1-e^{-p_{1} x}\right)+B\left(1-e^{-p_{2} x}\right)+C\left(1-e^{-p_{3} x}\right)+D\left(1-e^{-p_{4} x}\right)+E\left(1-e^{-p_{5} x}\right)+F\left(1-e^{-p_{6} x}\right)
$$

To determine the lower and upper limit of the search range for each desired coefficient, which is included in the dependence according to step 2. To determine the initial approximation for each desired coefficient, which is included in the dependence according to step 2. To search for dependencies using the Matlab fit () function. To perform the analysis of the obtained dependency on additional output parameters of combinations of descriptive statistics produced by the fit function, such as: Determination coefficient R2 (rsquare) and matched R2 (adjrsquare), sum of squared errors (sse) and mean square error (rmse). Then complete the analysis with the help of visual inspection and validation of the graphs of experimental data and data according to approximating dependence by plotting these graphs in the same axis.

The limits of the ranges in Section 3 can be selected from a range of positive integer values, for example, zero for the lower limit, and 1000 for the upper limit, or selected based on the values of 
the coefficients obtained during the first iteration of finding the approximation. The initial approximation for each desired coefficient entering the approximating dependence can be chosen randomly in the range from 0 to 1 , or the values found during the first iteration of finding the approximating dependence.

\section{RESULTS}

For HFSVC IC LM2676 in TO-220-7 case in the absence of an additional radiator for heat removal, the time dependence of the crystal temperature is obtained to (3).

$$
\begin{aligned}
T_{6}(t)= & 2,473\left(1-e^{-0,1794 t}\right)+1,125\left(1-e^{-0,9827 t}\right)+2,773\left(1-e^{-0,03683 t}\right)+ \\
& +24,39\left(1-e^{-0,002466 t}\right)+3,366\left(1-e^{-0,03761 t}\right)+23,3\left(1-e^{-0,006256 t}\right)
\end{aligned}
$$

Figure 1 shows the result of approximation by six exponentials. In the upper graph, the two dependencies experimental and approximating merged and are visually indistinguishable. The bottom graph shows the difference between these dependencies.

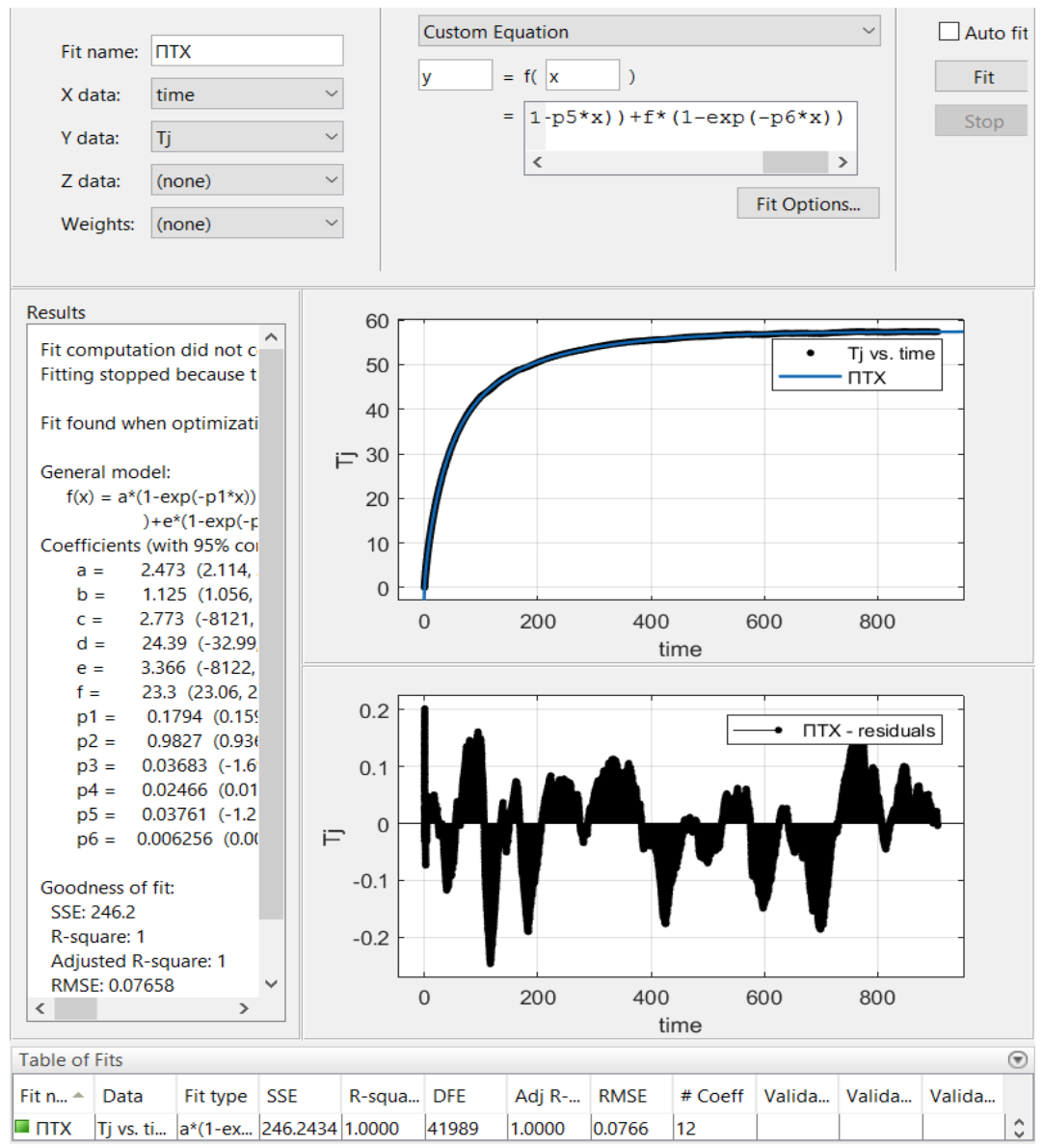

Figure 1. Graphical user interface of software toolkit in Matlab

The obtained analytical dependence agrees well with the experimental one. This difference in the whole range does not exceed $0.26^{\circ} \mathrm{C}$. According to the data of this equation, it is possible to construct a six-letter model in the form of the Foster scheme, in which the resistances are equal to the initial values of the exponentials, and the capacitances are determined by dividing the corresponding time constant by its resistance. So for the first $\mathrm{RC}$ element, we determine $\mathrm{R} 1=2.473 \mathrm{Ohm}, \tau=1 / \mathrm{p} \mid=1 / 0,1794=5,574 \mathrm{c}$, $\mathrm{C}=\tau / \mathrm{R} 1=13.79 \mathrm{~F}$.

It is important to note that it is impossible to unambiguously compare each resistance to some kind of physical transition (for example, crystal-crystal holder (die), etc.), since the heat fluxes inside and outside the microcircuit are parallel, and the model is represented by a series connection of links. The standard [16] 
and paper [14] also state that the FOSTER model "cannot be used to characterize the thermal structure. Real thermal capacitances are always connected to the base node-similarly to electrical "ground", since the stored thermal energy is proportional to the temperature of one node, and not the temperature difference of two nodes, which is determined by the FOSTER model'[16]. This is a significant drawback of the FOSTER model, although it is a fairly correct mathematical model of transitional behavior.

Similarly, approximations of four, three, two, and one exponent are obtained. These results are shown in Figure 2, in which the approximating dependence graph is shown in gray while the experimental data in black. Figure 2(a) shows the TTR approximation by four exponents. Figure 2(b) shows a version of the TTR approximation by three exponents. The obtained analytical dependences are in good agreement with the experimental ones. Mismatch graphs are visually indistinguishable. The temperature difference between the calculated and experimental TTR does not exceed $0.26^{\circ} \mathrm{C}$.

Figure 2(c) shows a variant of the TTR approximation by two exponents. The resulting analytical dependence $T_{2}(t)=24,64\left(1-e^{-0,0482 t}\right)+32,5\left(1-e^{-0,00793 t}\right)$ has a mismatch in the initial part of the experimental

TTR not more than $1,3^{\circ} \mathrm{C}$. Figure $2(\mathrm{~d})$ shows a variant of TTR approximation by one exponent. Visually dependencies mismatch. The resulting analytical dependence $T_{1}(t)=56,045\left(1-e^{-0,015 t}\right)$ has poor agreement with experimental results. The temperature difference between the calculated and experimental TTR in the initial part reaches $4.2^{\circ} \mathrm{C}$. A significant deviation of the approximating dependence on the experimental one is observed in the initial part of the TTR. For this part, you can perform an approximation again with two or three exhibitors as shown in Figure 3.
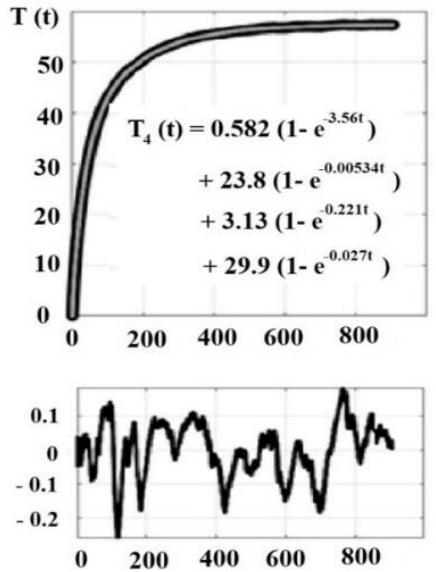

(a)
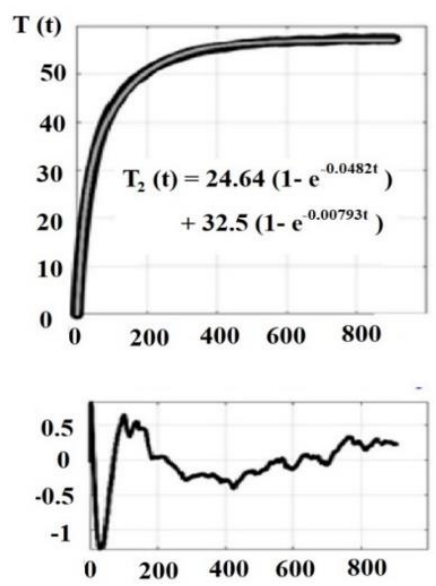

(c)
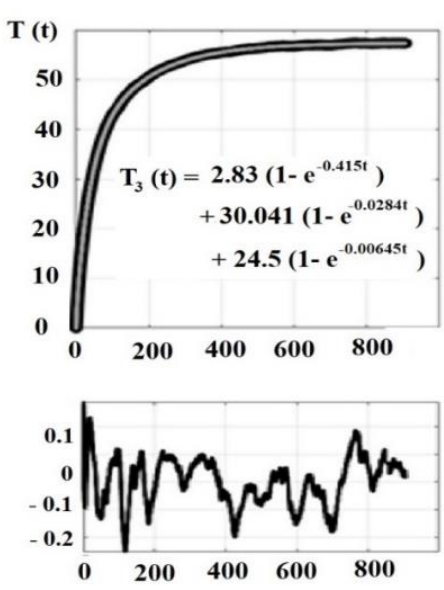

(b)
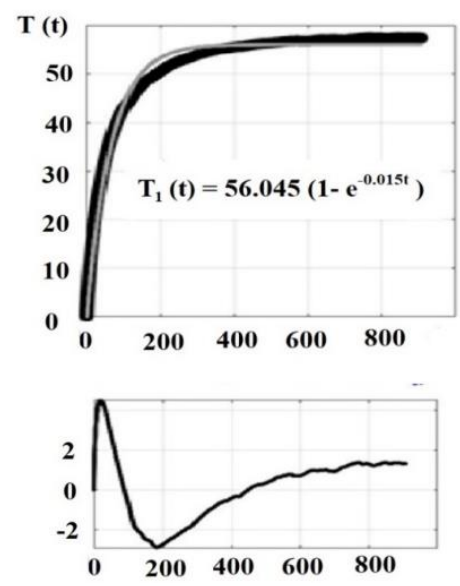

(d)

Figure 2. Approximation results by (a) Four exponents, (b) Three exponents, (c) Two exponents, (d) One exponent 
Figure 3(a) shows a variant approximation by three exponents. The resulting analytical dependence $T_{3}(t)=2,35\left(1-e^{-0,291 t}\right)+0,509\left(1-e^{-4,25 t}\right)+39,4\left(1-e^{-0,0264 t}\right)$ agrees well with the experimental results. The temperature difference between the calculated and experimental TTR does not exceed $0.05^{\circ} \mathrm{C}$. This temperature difference is shown in the lower graphs of Figure 3. Figure 3(b) shows a variant of approximation by two exponents. The resulting analytical dependence $T_{2}(t)=1,03\left(1-e^{-1,503 t}\right)+28,9\left(1-e^{-0,0476 t}\right)$ agrees well with the experimental results. The temperature difference between the calculated and experimental TTR does not exceed $0.13^{\circ} \mathrm{C}$.
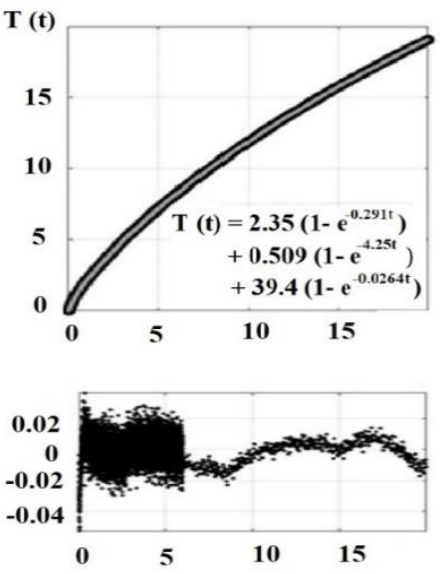

(a)
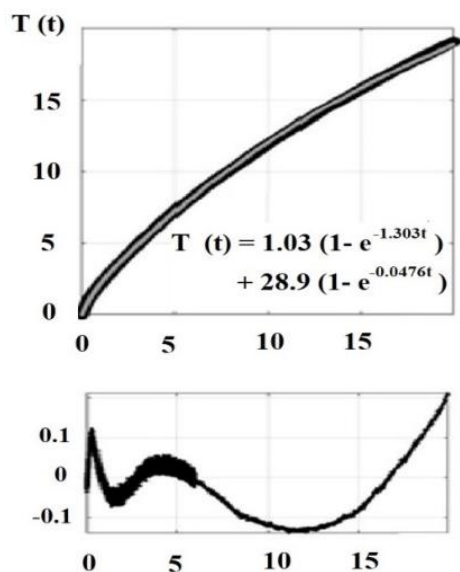

(b)

Figure 3. Approximation results for the initial TTR par, (a) Variant approximation by three exponents,

(b) Variant of approximation by two exponents

It should be noted that the dependences for the initial TTR area have exponents that are significantly different from the total TTR exponent. One of the exponents has a time constant of less than one second, which characterizes the rapid change in temperature. On the basis of the obtained analytical dependencies, if necessary, it is possible to construct electrical models in the form of chains of RC elements in which the change in voltage $\mathrm{U}(\mathrm{t})$ during the transient process will coincide numerically with the TTR. Foster multilink schemes are often used, in which each exponent corresponds to a link consisting of parallelconnected $\mathrm{R}$ and $\mathrm{C}$. In this case, the initial value of the exponent determines the resistance $\mathrm{R}$ and the time constant $\tau=1 /|p|$ determines the capacitance $C=\tau / R$.

Figure 4 shows the thermoelectric chip model, in which the current source $\mathrm{J}$ simulates the heating power $\mathrm{P}$ (thermal losses), the potentials at the nodes of circuit 1 and 0 simulate the temperature of the UJ p-n junction the ambient temperature Ta, the EMF source E imitates ambient temperature Ta. In this case, the total temperature of the $\mathrm{p}-\mathrm{n}$ junction $\mathrm{TJ} \Sigma(\mathrm{t})=\mathrm{Ta}+\mathrm{TJ}(\mathrm{t})$ contains the constant component $\mathrm{Ta}$ and the variable $\mathrm{TJ}(\mathrm{t})$, due to heating. To simplify writing in the following equations, only the variable component of the temperature $\mathrm{TJ}(\mathrm{t})$ due to heating is considered.

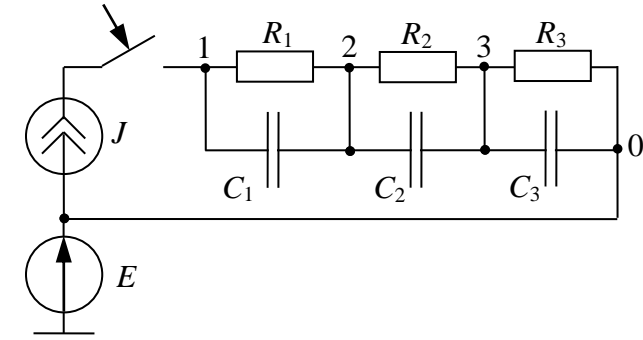

(a)

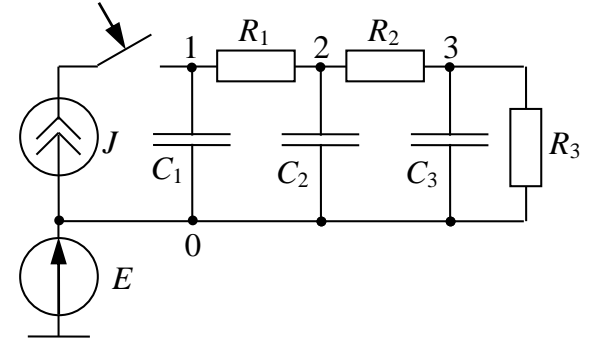

(b)

Figure 4. Representation of the electric-thermal model by, (a) The fosterdiagram, (b) The cauer 
Obviously, one should not look for a correspondence between electrical and thermal resistances. Resistances R1, R2, R3 are calculated. Together with the capacitances C1, C2, C3 they provide a change in the voltage $U(t)$ during the transient process (when the current source $J$ is connected) numerically coinciding with the change in the crystal temperature (TTR) of the microcircuit. The resulting thermoelectric model can be implemented in any program for modeling electrical circuits (Orcad, Multisim, Matlab/Simulink, etc.) and investigate various transient conditions with regard to thermal processes. The voltage changes over time U1 (t) will correspond to similar changes in the crystal temperature of the microcircuit $T(t)$.

In addition, the differences in the TTR circuits experimentally obtained in the heating mode of the sample and in the cooling mode, with the same thermal power, were also analyzed. These TTRs match quite well. The maximum discrepancies of the graphs were at a time level of $100 \mathrm{~s}$, on the order of 1-4 for various suitable samples. For other time intervals of the same TTR there are even better coincidence of the graphs.

Figure 5 shows as an example, an oscillogram of the passage of an electric pulse on a thermal electric model. Considering the oscillogram of the electrical transient process with a short-term change in the magnitude of the current source $\mathrm{J}$, we can estimate the nature of the transition thermal process with a corresponding change in the heating power P. Thus, with the passage of a current pulse of $2 \mathrm{~J}$ (double value) with a pulse duration of $60 \mathrm{~s}$, the voltage U1 changed from $57 \mathrm{~V}$ to $128 \mathrm{~V}$, which corresponds to a change in the temperature of additional heating of the HFSVC crystal from $57^{\circ} \mathrm{C}$ to $128^{\circ} \mathrm{C}$. At the same time, the total crystal temperature for an ambient temperature of $20^{\circ} \mathrm{C}$ will change from $77^{\circ} \mathrm{C}$ to $148^{\circ} \mathrm{C}$, which is a value close to the threshold value of the response of the temperature protection of the IMS VIP. A return to the nominal temperature of $57^{\circ} \mathrm{C}$ lasts about $1000 \mathrm{~s}$.

To verify the correctness of the model, a physical experiment was performed. The microcircuit was heated through a power diode for 5 minutes and on the free $\mathrm{p}-\mathrm{n}$ junction the forward voltage UF was determined, which was later converted to the temperature TJ. The obtained dependence TJ (t) coincides well with the graph in Figure 5.

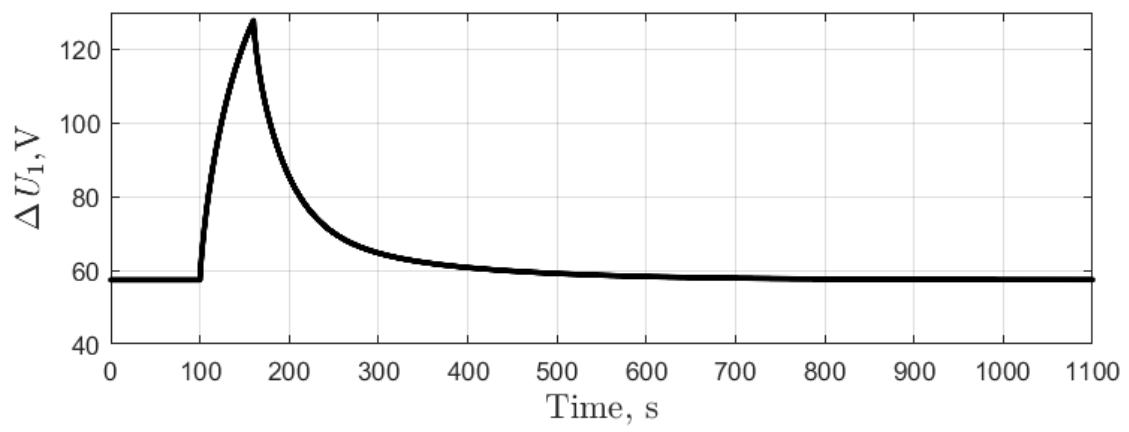

Figure 5. The passage of heat pulse on the model

\section{DISCUSSION}

When forming the TTC electric-thermal model, the most important is the choice of the model structure, which in the problem under consideration corresponds to the determination of the number of exponents. In this case, it is often difficult to obtain an approximating dependency well coinciding with the TTC initial area. To reduce the influence of these factors when obtaining an approximating dependence, it is possible to use the spectraldensity of time constants (SDTC) [16, 19, 20, 23].

In $[11,20,23,25]$ it is proposed to do this by sampling a continuous graph of the spectral density of time constants with a fixed step dz. This, in turn, leads to an increase in the number of exponents and complication of the model, while simultaneously increasing the difficulty of calculation associated with the use of numbers with increased accuracy (double precision of numbers of the standard double float type with small $\mathrm{dz}$ is often not enough).

The authors propose to use SDTC to determine the number of exponents, and to specify initial approximations when searching for constant time (coefficients at the exponent degree). The SDTC graphs of the experimental TTR, obtained by heating the LM2676 chip, is shown in Figure 6. The graph of the most important initial part of the structural function, obtained on the SDTC bas is is shown in Figure 7.

From the graph in Figure 6, four extremums are visible, which allows us to choose a model with four exponents. Additionally SDTC is a reliable source of information for specifying the initial approximation of the coefficients of exponent degrees, for which the choice of the initial approximation 
and search ranges is most important for obtaining qualitative results in the approximation. As it can be seen from the SDTS graph, shown in Figure 6, it clearly identifies four time constants, the value of which practically coincides with the results of the approximation shown in Figure 2(a).

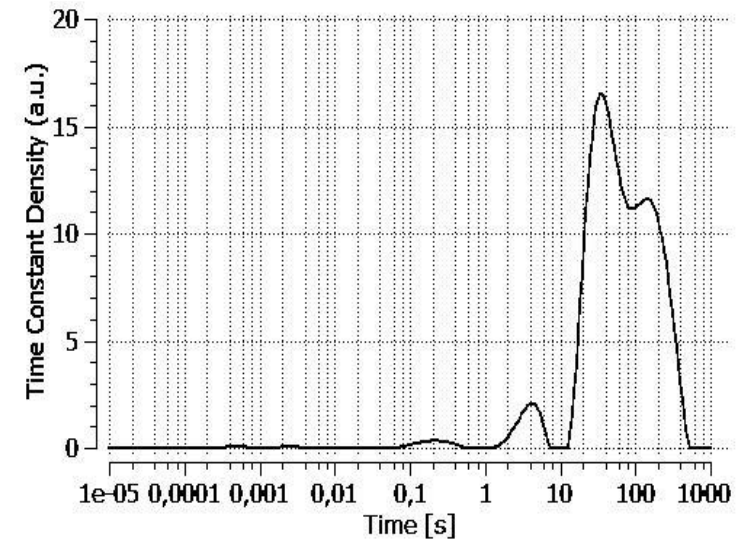

Figure 6. Graph of the spectral density of time constants

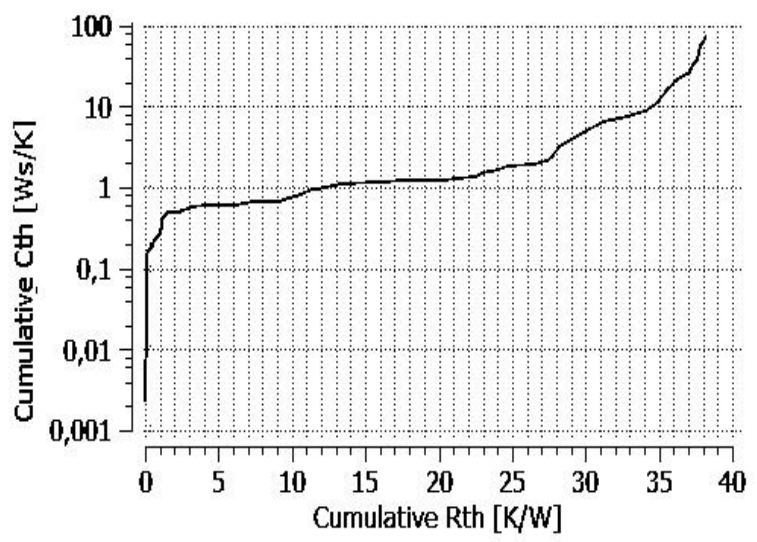

Figure 7. Structure function graph

Due to the initially inaccurate adjustment of the filters of the reverse convolution operation and the initial data of the experimentally determined TTR, the quality schedule of the SDTC is difficult to obtain at the first iteration. This is manifested in the fact that extremes can be shifted, which prevents you from immediately getting good approximation results (especially for the TTR initial part), or lowering the dimension of the problem, by reducing the number of desired coefficients in the approximation by eliminating constant time. The graph in Figure 6 was obtained by an iterative method of adjusting the deconvolution operation filter.

In the method of approximation used in this work, the SDTC graph is used only to specify the initial approximations and ranges of the search for coefficients with exponential powers of the approximation dependence. This significantly improved the quality of the approximation while simultaneously reducing the number of exponents included in the approximating relationship because from the graph of the structural function as shown in Figure 7, the minimal set of these exponents does not explicitly follow and there always exists the probability of its inaccuracies due to filtering, as it has been mentioned already. This allows to obtaina minimized model of the thermal process in the IC without significant losses in the degree of its reliability.

\section{CONCLUSION}

The analysis of the methods for obtaining the thermoelectric IC model showed the main difficulties of their application for building models with a minimized number of RC circuits. The proposed solution is to refine the minimum structure of the thermoelectric model both on the basis of direct iteration based on the least squares method using the Levenerg-Marquardt optimization algorithm as well as using the SDTC graph as initial data, which allows reducing the influence of the filter factor in the deconvolution operation, with building a thermal model using the TTR structure function. The results obtained can be used to build IC behavioral models, taking into account the thermal processes occurring in them.

\section{ACKNOWLEDGMENTS}

The work was carried out with the financial support of the Russian Federation Ministry of Education and Science (project No. 8.1729.2017/4.6). 


\section{REFERENCES}

[1] M. Galicia, P. Zajac, C. Maj and A. Napieralski, "Characterization of thermal vias for 3D ICs using FEM analy sis," 2015 21st International Workshop on Thermal Investigations of ICs and Systems (THERMINIC), Paris, pp. 1-4, 2015.

[2] Y. C. Gerstenmaier, W. Kiffe and G. Wachutka, "Combination of thermal subsystems modeled by rapid circuit transformation," 2007 13th International Workshop on Thermal Investigation of ICs and Systems (THERMINIC), Budapest, pp. 115-120, 2007.

[3] Z. Zhou, P. M. Holland and P. Igic, "Compact thermal model of a three-phase IGBT inverter power module," 2008 26th International Conference on Microelectronics, Nis, pp. 167-170, 2008.

[4] A. Y. Tang, et al., "Steady-state and transient thermal analysis of high-power planar Schottky diodes," In 22nd International Symposium on Space Terahertz Technology, 2011.

[5] V. Kosel, R. Sleik and M. Glavanovics, "Transient non-linear thermal FEM simulation of smart power switches and verification by measurements," 2007 13th International Workshop on Thermal Investigation of ICs and Systems (THERMINIC), Budapest, pp. 110-114, 2007.

[6] J. E. Standard, "Compact Thermal Model Overview," JESD15-1, JEDEC Solid State Technology Association, 2008.

[7] V. Guerrero, "Behavioral modelling and identification of power electronics converters and subsystems based on transient response: Ph.D. dissertation," Madrid, Spain: Departament of Electronic Technology, Charles III Universi-ty of Madrid, pp. 447, 2013.

[8] D. L. Osipov, "The use of Behavioral Models for the Design of Complex-Functional Blocks of Analog-to-Digital Aonverters," Dis. PhD Eng. Sci. Moscow, 2013.

[9] A. N. Shkolin, A. Y. Drakin and V. F. Zotin, "Development of automated measurement systems for testing integrated circuits of switching converters," 2018 Moscow Workshop on Electronic and Networking Technologies (MWENT), Moscow, pp. 1-4, 2018.

[10] MIL-STD-883E. Method 1012.1, "Thermal Characteristics of Integrated Circuits," pp. 13, 1980.

[11] EIA/JEDEC JESD51-1 standard. "IC Thermal Measurement Method-Electrical Test Method (Single Semiconductor Device)." Arlington, USA: Electronic Industries Alliance, pp. 33, 1995.

[12] V. A. Sergeev, "Tetenkin Ya. G. Algorithm for determining the thermal pa-rameters of digital integrated circuits by transient thermal characteristics," Automation of Control Processes, vol. 1, pp. 112-119, 2016.

[13] M. I. Gorlov, et al., "Measurement of the noise parameters of semiconductor devices," Measurement Techniques, vol. 49, pp. 1241-1245, 2006

[14] J. Altet, et al., "Thermal testing of integrated circuits," New York; London: Springer, 2011.

[15] K. Górecki and J. Zarebski, "Nonlinear Compact Thermal Model of Power Semiconductor Devices," in IEEE Transactions on Components and Packaging Technologies, vol. 33, no. 3, pp. 643-647, Sept 2010.

[16] S. K. Ravindran, et al., "A self-sustaining micro thermomechanic-pyroelectric generator," Applied Physics Letters, vol. 99, pp. 104102, Sep 2011.

[17] JEDEC JESD51-14 standard, "Transient Dual Interface Test Method for the Measurement of the Thermal Resistance Junction to Case of Semicon-ductor Devices with Heat Flow through a Single Path," Arlington, USA: JEDEC Solid State Technology Association, pp. 46, 2010.

[18] A. Poppe, et al., "Thermal measurement and modeling of multi-die packages," IEEE Transactions on components and packaging technologies, vol. 32, pp.484-492. 2009.

[19] V. Székely, et al., "THERMODEL: A tool for thermal model generation, and application for MEMS," Analog Integrated Circuits and Signal Processing, vol. 1, pp.49-59, 2001

[20] V. Székely and T. Van Bien, "Fine structure of heat flow path in semiconduc-tor devices: A measurement and identification method," Solid-State Elec-tron.vol. 31.pp. 1363-1368, 1988.

[21] D. Schweitzer, H. Pape and L. Chen, "Transient Measurement of the Junction-To-Case Thermal Resistance Using Structure Functions: Chances and Limits," 2008 Twenty-fourth Annual IEEE Semiconductor Thermal Measurement and Management Symposium, San Jose, CA, pp. 191-197, 2008.

[22] H. Pape, D. Schweitzer, L. Chen, R. Kutscherauer and M. Walder, "Development of a standard for transient measurement of junction-to-case thermal resistance," 2011 12th Intl. Conf. on Thermal, Mechanical \& MultiPhysics Simulation and Experiments in Microelectronics and Microsystems, Linz, pp. 1/8-8/8, 2011.

[23] V. Székely and A. Szalai "Measurement of the time-constant spectrum: Sys-tematic errors, correction," Microelectron. J. vol. 43, pp. 904-907, 2012.

[24] M. A. Huque, L. M. Tolbert, B. J. Blalock and S. K. Islam, "Silicon-on-insulator-based high-voltage, hightemperature integrated circuit gate driver for silicon carbide-based power field effect transistors," in IET Power Electronics, vol. 3, no. 6, pp. 1001-1009, Nov. 2010.

[25] V. Szekely, "Identification of Networks by Deconvolution: Chances and Limits," IEEE Trans. CIRCUITS Syst, vol. 45 pp. 15,1998 .

[26] A. L. Zakgeim, A. E. Chernyakov, A. S. Vaskou, V. K. Kononenko and V. S. Niss, "Comparative analy sis of the thermal resistance profiles of power light-emitting diodes cree and rebel types," 2013 14th International Conference on Thermal, Mechanical and Multi-Physics Simulation and Experiments in Microelectronics and Microsystems (EuroSimE), Wroclaw, pp. 1-7, 2013.

[27] V. S. Niss, et al., "Estimation of thermal parameters of power bipolar transistors by the method of thermal relaxation differential spectrometry," Devices and Methods of Measurements, vol. 6, pp. 249-256, 2015.

[28] Y. A. Bumai, et al., "Relaxation impedance spectrometer of thermal processes," Elektronika info, vol. 3, pp. 58-59, 2010. 
[29] A. S. Vaskou, et al., "Diagnostics of the technological characteristics of high-power transistors using relaxation impedance spectrometry of thermal processes," Russian Microelectronics, vol. 44, pp. 579-584, 2015.

[30] R. I. Ivanovsky, "Approximations of observational data in the Mathcad Pro environment," Scientific and Practical Journal ExponentaPro Mathemat-ics In Applications, 2003.

\section{BIOGRAPHIES OF AUTHORS}

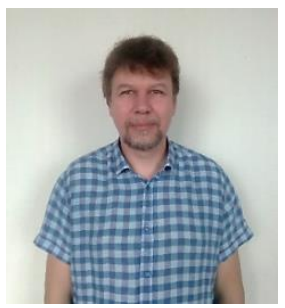

Drakin A. Yu. is a Candidate of Engineering Sciences (Ph.D.), an Associate Professor of the department of Industrial Electronics and Electrical Engineering of Bryansk State Technical University. He is a Senior Researcher of the Research Laboratory of ATM. His research areas are Electric Drive and Automation of Industrial Plants and Technological Complexes.

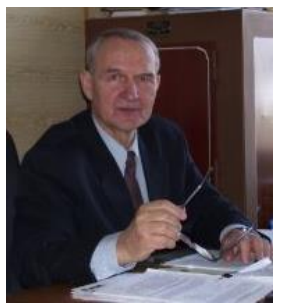

L. A. Potapov is a Head of the Department "Industrial Electrical Engineering and Electronics", Professor, Doctor of Technical Sciences of Bryansk State Technical University. Stages of his work biography: 1961-1971, Electromechanical Research Institute, Voronezh (Engineer, Head of the Group, Head of the laboratory); 1971-2002, Bryansk Institute of Transport Engineering, Associate Professor; 2002-present, Bry ansk State Technical University, Doctor of Engineering, Professor, Head of the Department "Industrial Electrical Engineering and Electronics." His research areas are Test automation, Simulation of Electromechanical Devices based on the Field of Theory.

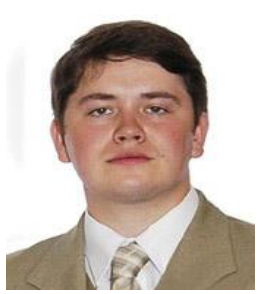

A. N. Shkolin is a Researcher and Senior Lecturer of the Department "Electronic, RadioElectronic and Electrotechnical Systems" of Bryansk State Technical University. In 2003 he graduated from BSTU with a degree in Industrial Electronics. From June 2003 to November 2003 he worked as an electronic engineer at the Automated Electric Drive Department. From November 2003 to October 2006, a graduate student of the department "Automated Electric Drive", at the same time as a graduate student, worked as an assistant and an electronic engineer of the department. Currently, he works as an assistant at the Department of Electronic, Radio-Electronic and Electrical Systems. His research areas are Mathematical modeling and study of the dynamics of automated heat supply systems. 